\title{
Présence chez un Rongeur du Chili d'un Nématode Inglamidinae (sub. fam. nov.) appartenant aux Amidostomatidae, famille connue des Mammifères d'Australie
}

\author{
par Marie-Claude DURETTE-DESSET *, M. Abékoué DENKE * \\ et R. MURUA ** \\ * Laboratoire de Zoologie (Vers) associé au C.N.R.S., \\ Muséum national d'Histoire naturelle, 43, rue Cuvier, F 75231 Paris Cedex 05. \\ ** Instituto de Ecologia y Evolucion, \\ Casilla 57-D, Universal Austral de Chile, Valdivia, Chile
}

\section{Résumé.}

Une nouvelle sous-famille d'Amidostomatidae est décrite chez des Cricétidés du Chili : Inglamidinae n. sous-f., représentée par le genre type et l'espèce type: Inglamidum akodon n. gen., n. sp.

La sous-famille est caractérisée, d'une part, par des éléments archaïques (structure céphalique), qui la rattachent aux autres Amidostomes, et, d'autre part, par des éléments évolués (monodelphie, synlophe, spicules), qui la rattachent aux Héligmosomes et, en particulier, à la lignée Viannaia-Viannella, parasite de Marsupraux sud-américains.

Nous interprétons cette espèce comme un parasite de capture et nous pensons que des formes proches doivent se trouver chez d'autres Mammifères endémiques, en particulier les Marsupiaux. Du point de vue paléobiogéographique, cela permettrait de situer l'origine des Amidostomatidae de Mammifères à l'ère secondaire, au moment de l'expansion des Marsupiaux. Ces Nématodes auraient alors donné naissance aux autres Trichostrongyloidea par atrophie de la capsule buccale et aux Ancylostomatoidea par élaboration progressive des structures buccales. Contrairement à Inglis, nous préférons considérer les genres Globocephaloides et Hypodontus respectivement comme des Globocephalinae et Uncinariinae (Ancylostomatidae), et non comme des Amidostomatidae. 


\section{Summary.}

A chilean Rodent as host of a Nematode Inglamidinae (sub-fam. nov.) belonging to the Amidostomatidae previously known excluvisely from australian Mammals.

The Inglamidinae n. sub-fam., a new sub-family of Amidostomatidae from chilean Cricetidae is described with Inglamidum akodon gen. et sp. n. as the type genus and species.

Out of the 23 Akodon captured in the same area, three samples of this nematode have been collected from two different species. These findings confirm that we are dealing with a well-adapted parasite and exclude the possibility of a fortuitous catch or an accidental transfer.

This family displays two significant groups of taxonomic characters, including archaic characters such as cephalic structures which unite them to the Amidostomatidae, and on the other hand some more recently elaborated characters such as monodelphism and shape of synlophe and spicules which relate them to the Heligmosoms and more significantly to the line Viannaia-Viannella parasite of South-American Marsupials.

We interpret this species as a «parasite of capture» and we assume that very similar species might occur in other endemic Mammals, mostly Marsupials.

On a paleobiogeographical point of view this interpretation would make due allowance for postulating that the Amidostomatidae from Mammals have originated during the Secondary, concurrently with the Marsupial expansion.

These ancestral Nematodes would have given birth to the other Trichostrongyloidea through reduction of buccal cavity, and to the Ancylostomatoidea by further elaboration of buccal apparatus.

Contrary to Inglis's hypothesis we are in favour of the genera Globocephaloides and Hypodontus to be assigned to the Globocephalinae and Uncinariinae (Ancylostomatidae) respectively, rather than to the Amidostomatidae.

The occurrence of an Amidostomatidae in a South American Cricetidae is somewhat quite unexpected, mostly because this family is known to occur from australian Mammals only and alco because the parasited Mammals are the most primitive of the group (Monotremes and Marsupials).

La présence d'un Amidostomatidae chez des Cricétidés sud-américains peut paraître, à première vue, très inattendue, d'une part parce que cette famille est connue chez les Mammifères uniquement en Australie, d'autre part parce que les Mammifères parasités en Australie sont parmi les plus primitifs de leur groupe (Monotrèmes, Marsupiaux).

Sur 23 Akodon piégés dans la même région, le Nématode est retrouvé 3 fois, chez 2 espèces différentes ( $A$. olivaceus et $A$. sanborni). Il ne s'agit donc pas d'un parasite accidentel ou d'un transfuge, mais d'un véritable parasite de capture. 


\section{- Inglamidum akodon $\quad$ n. gen., n. sp.}

MATÉRIEl tyPe: 1 ơ, 2 \%, M.N.H.N. nº 983 HA.

Hôte : Akodon olivaceus ?.

LOCALISATION: Intestin grêle.

Origine GÉOGRAPHIQUe: San Martin, Province de Valdivia, 29-11-1974.

Autre matériel : Chez le même hôte (ơ), 2 ơ, 3 q piégés le 10-12-1974 à Esc. Normal, Province de Valdivia ( $\mathrm{n}^{\circ} 993$ HA).

- Chez un Akodon sanborni ơ, 1 ơ, 4 ㅇ piégés le 7-1-1975 à la Picada, Province d'Osorno (n ${ }^{\circ} 974$ HA).

\section{Description.}

Petits Nématodes enroulés de façon senestre le long de leur ligne ventrale, selon 2 à 5 tours de spire.

TÊTE : Elle est entourée par une vésicule céphalique. La bouche est soutenue par une capsule buccale haute de $10 \mu$, dont le diamètre est de $5 \mu$ à l'apex et de 12 à $14 \mu$ à la base. Présence de 3 dents: la dorsale est haute de $7 \mu($ ( ) $), 6,5 \mu\left(\delta^{*}\right)$, les latéro-ventrales sont hautes de $4,5 \mu$.

En vue apicale, la tête porte 6 lèvres. Seules les lèvres latérales restent individualisées. Les 4 autres lèvres fusionnent 2 à 2 (fig. $1, A$ ). Le long du bord extérieur des lèvres apparaissent les 6 papilles labiales internes portées par 6 pédoncules (fig. $1, E$ ). Ces pédoncules soutiennent également les amphides, les papilles du cycle labial externe et les 4 papilles céphaliques. Nous n'avons observé pour le cycle labial externe que les 4 papilles proches des céphaliques (fig. $1, B, C, D$ ).

SYNLOPHE: Dans les 2 sexes, le corps est parcouru par des arêtes cuticulaires au nombre de $29 \mathrm{chez}$ le $\delta$ et de $24 \mathrm{chez}$ la ㅇ. Chez d'autres spécimens $\delta$ examinés, le nombre d'arêtes cuticulaires varie entre 21 et 23 . Les arêtes débutent sur le bord postérieur de la vésicule céphalique pour la face latérale droite et à différents niveaux le long du champ latéral gauche (fig. 2, C). Chez le $\delta$, les arêtes disparaissent à $220 \mu$ en avant de la bourse caudale et à $80 \mu$ en avant de la vulve chez la $q$.

En coupe transversale, la pointe des arêtes est orientée de la droite vers la gauche. Les arêtes ventrales sont légèrement plus fortes que les autres arêtes (fig. 2, A).

MÂle: Chez un $\delta^{\widehat{t}}$ long de $3,2 \mathrm{~mm}$ et large de $80 \mu$ dans sa partie moyenne, la vésicule céphalique est haute de $83 \mu$ sur $30 \mu$ de large. Anneau nerveux, pore excréteur et deirides situés respectivement à $220 \mu, 285 \mu$ et $305 \mu$ de l'apex. CEsophage long de $420 \mu$ (175 $\mu$, œsophage musculaire, $245 \mu$, œsophage glandulaire) (fig. 2, B).

Bourse caudale légèrement asymétrique, avec un lobe droit plus développé. Lobe dorsal bien marqué. Côte dorsale courte, divisée en 2 rameaux eux-mêmes bifurqués. Côtes 8 longues et grêles. Côtes 4 plus courtes que les 5 adjacentes (fig. 2, E, F). Cône génital mal observé. Gubernaculum en forme de fer de lance haut de $60 \mu$ sur $10 \mu$ de large à sa base. 
Spi-ules sub-égaux, ailés, longs de $145 \mu$, dont $45 \mu$ pour le manche. Leur extrémité postérieure est arrondie (fig. 2, G).

Femelle: Chez une $q$ longue de $1,4 \mathrm{~mm}(1)$, large de $80 \mu$ dans sa partie antérieure et de $100 \mu$ dans sa partie postérieure, la vésicule céphalique est haute de $60 \mu$ sur $40 \mu$ de large. Anneau nerveux, pore excréteur et deirides situés à $145 \mu, 170 \mu$ et $185 \mu$ de l'apex. Esophage long de $415 \mu$.

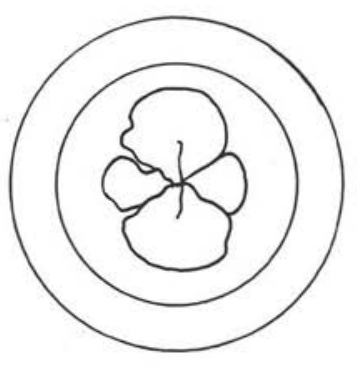

A

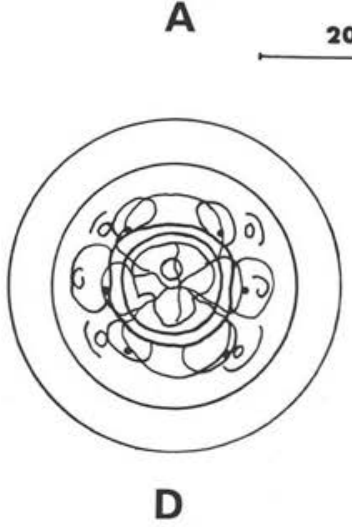

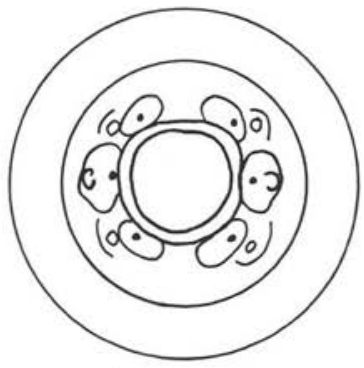

B

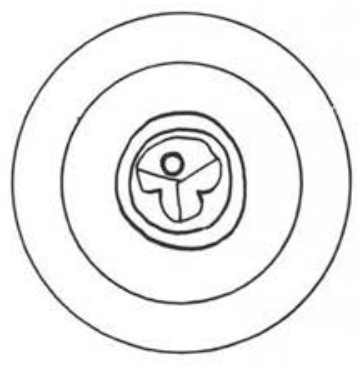

C

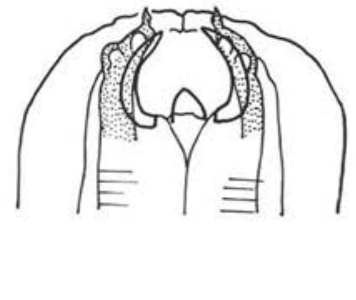

E

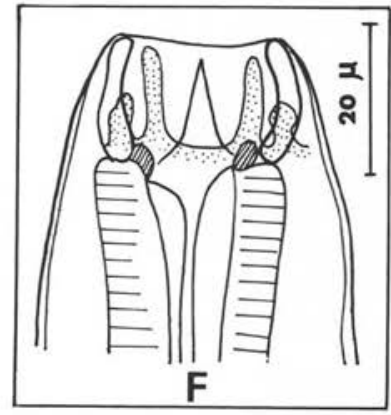

FIG. 1. - Inglamidum akodon n. sp. A, B, C, $q$, tête en vue apicale, vue successivement au niveau des lèvres, au niveau des papilles, au niveau des dents ; $\mathrm{D}$, id., ensemble de tous les niveaux reconstitué ; E, tête en vue dorsale. - Amidostomum fulicae (Rudolphi, 1819); F, + , tête en vue dorsale. (D'après Wertheim et Durette-Desset, 1976).

L'appareil génital peut être considéré comme monodelphe, bien qu'il existe une branche génitale postérieure, atrophiée, qui mesure $50 \mu$. La vulve s'ouvre à $120 \mu$ de la pointe caudale. Vagina vera long de $30 \mu$. Vestibule et sphincter longs respectivement de $110 \mu$ et de $50 \mu$ sur $62 \mu$ de large. La trompe forme une boucle, mais sa longueur exacte n'a pu être précisée. Utérus mesurant $370 \mu$ et contenant 9 œufs non embryonnés, hauts de $60 \mu$ sur $50 \mu$ de large (fig. 2, D).

Queue longue de $40 \mu$, portant une pointe caudale de $10 \mu$ (fig. 2, D).

(1) Nous avons choisi une $\subsetneq$ jeune pour la description, car, chez les $q$ âgées (qui atteignent jusqu'à $3,5 \mathrm{~mm}$ ), l'utérus grossit, se superpose à l'ovéjecteur, qui devient difficile à observer. 


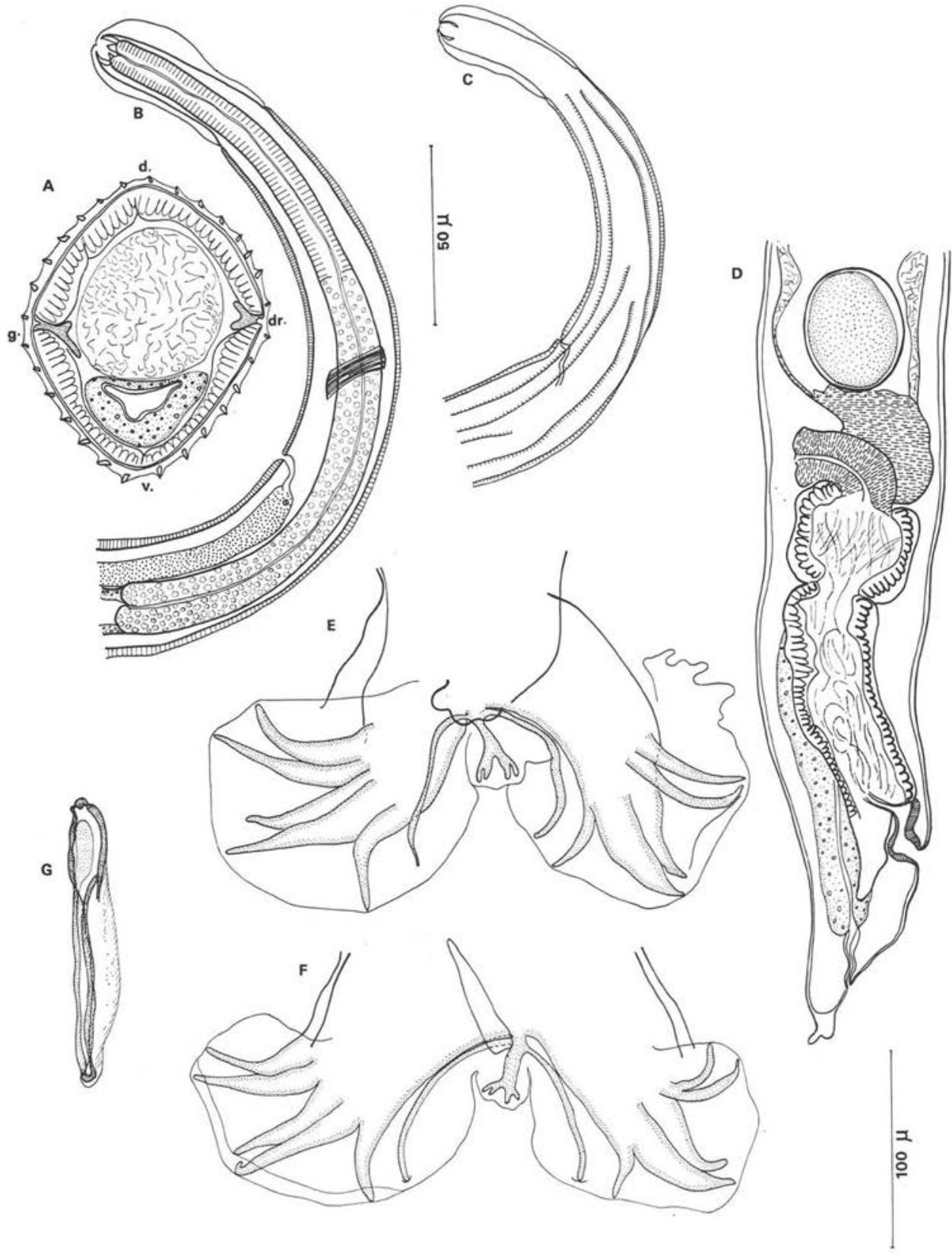

FIG. 2. - Inglamidum akodon n. sp. A, $\delta^{\lambda}$, coupe transversale au milieu du corps; B, $\delta$, extrémité antérieure, vue latérale gauche; C, id., montrant la naissance des arêtes cuticulaires; D, ㅇ, extrémité postérieure, vue latérale droite; E, ố, bourse caudale étalée, mais déchirée, vue ventrale; $F$, autre spécimen, $\delta^{*}$, bourse caudale, vue ventrale; $G, \delta^{2}$, spicule disséqué.

A, éch. : $50 \mu$; B à $\mathrm{G}$, éch. : $100 \mu$. 


\section{Discussion}

\section{I. - Analyse morphologique.}

La structure céphalique de cette espèce est caractéristique de celle des Amidostomatinae, tels que ceux-ci sont définis par Inglis, 1967. L'existence de 6 pédoncules verticaux, extérieurs à la capsule buccale et contenant les filets nerveux (comparables à la structure classique des Kalicéphales) est décrite par Inglis chez Globocephaloides, mais elle existe également, de façon plus discrète, dans le genre Amidostomum lui-même (cf. Wertheim et Durette-Desset, 1976).

A côté de cette structure céphalique très caractéristique, qui permet de préciser les affinités de l'espèce, les autres caractères essentiels sont: 1) La monodelphie. 2) Le synlophe, dont nous ne connaissons pas d'équivalent. Etant formé d'arêtes petites, nombreuses et sub-égales, il ressemble à celui de toutes les formes spécialisées, Stilestrongylus par exemple. Mais l'axe d'orientation des arêtes est strictement frontal, ce qui le distingue des Nippostrongylinae, et laisse supposer une hyperévolution de la lignée Viannaia-Viannella (cf. Durette-Desset, 1968). 3) Les spicules. Ils sont courts et simples, à extrémité arrondie, et évoquent ceux de certaines espèces du genre Viannella (cf. Durette-Desset, 1971).

Ainsi, comme chez la plupart des espèces reliques, il y a coexistence de caractères phylétiques primitifs (structure céphalique), qui rattachent indiscutablement l'espèce aux Nématodes connus chez les Marsupiaux australiens, et de caractères plus adaptatifs, souvent hyperspécialisés (monodelphie, synlophe, spicules), qui évoquent, au contraire, des affinités avec les Marsupiaux et même les Caviomorphes sud-américains.

\section{II. - Intérêt phylétique de l'espèce.}

Dans son travail fondamental, Inglis, 1967, montre que les Amidostomatidae, en dehors du genre Amidostomum, parasite cosmopolite d'Oiseaux, et du genre Amphibiophilus, parasite de Reptiles et d'Amphibiens en Afrique et en Chine, sont constitués par de nombreux genres exclusivement australiens. Il démontre en outre que Globocephaloides, d'une part, et Hypodontus, d'autre part, dérivent vraisemblablement de façon directe des Amidostomes et les place dans cette famille. La découverte d'un Amidostome typique chez un Mammifère, en dehors de l'Australie, rend nécessaire un nouvel examen du problème.

L'Australie pourrait être, en réalité, la région du monde où les Amidostomes ont pris leur plein développement, mais pas forcément leur naissance.

$\mathrm{Si}$ nous sommes en accord avec Inglis, lorsqu'il montre que Globocephaloides et Hypodontus dérivent vraisemblablement des Amidostomes, nous ne pensons pas que ces deux genres soient totalement différents respectivement des Globocephalinae et des Uncinariinae. 
Les Amidostomes, bien que presque partout éteints, sont, à l'origine, cosmopolites, et non exclusivement australiens, et nous pensons que les Globocephalinae, puis les Uncinariinae, dérivent tous des Amidostomes, aussi bien en Australie que dans le reste du monde.

Contrairement à Inglis, nous excluons donc ces 2 genres (Globocephaloides et Hypodontus) des Amidostomatidae.

\section{III. - Intérêt paléobiogéographique de l'espèce.}

L'espèce décrite est parasite d'un Rongeur Cricétidé, mais il s'agit certainement d'un phénomène de capture. Les Cricétidés ont envahi la région néotropicale à la fin du Tertiaire. Le Nématode relique parasite de l'Akodon appartient à une lignée très ancienne. Des recherches complémentaires montreront presque certainement l'existence de Nématodes plus primitifs de la même lignée, soit chez des Reptiles-Oiseaux, soit, plus vraisemblablement, chez des Marsupiaux ou d'autres Mammifères endémiques.

L'analogie très poussée des structures céphaliques entre la faune australienne et l'espèce américaine rend un phénomène de convergence peu vraisemblable, et nous ne pensons pas que l'adaptation de la lignée se soit effectuée indépendamment, à deux reprises, depuis les Reptiles-Oiseaux jusqu'aux Mammifères. L'hypothèse la plus plausible nous paraît être l'existence d'un Nématode ancestral chez les Marsupiaux à l'époque où ceux-ci sont passés d'Amérique en Australie.

Il n'existerait plus actuellement, chez les Mammifères, que des reliques, rares et localisées à la Côte Est de l'Amérique du Sud, ou, au contraire, très largement diversifiées chez les Marsupiaux australiens. Ce serait donc à l'ère secondaire qu'il faudrait situer l'origine des Amidostomes, ce qui est très satisfaisant, car, d'un point de vue morphologique, ils peuvent avoir donné naissance aussi bien à l'ensemble des Trichostrongyloidea, par atrophie de la capsule buccale, qu'aux Ancylostomatoidea, par élaboration progressive des structures buccales.

En conclusion, ces Nématodes ne peuvent être rapprochés que des Amidostomatinae, tels qu'ils sont définis par Inglis. Ces parasites ne comprenaient jusqu'à maintenant que des parasites australiens, ainsi que deux genres cosmopolites et un genre chez les Amphibiens et les Reptiles en Afrique et en Chine. Il s'agit donc du premier Amidostome de Mammifères connu hors de l'Australie. Bien que la structure céphalique soit tout à fait conforme à celle des Nématodes parasites de Mammifères australiens, tous les caractères accessoires (monodelphie, synlophe, spicules) sont d'un type assez caractéristique de la lignée des Trichostrongylides de Marsupiaux sud-américains.

Nous pensons qu'il est nécessaire non seulement de créer un genre, mais de classer ce genre dans une sous-famille particulière à côté de celle des Amidostomatinae. 


\section{Inglamidinae n. s/fam.}

Amidostomatidae; cavité buccale globulaire avec 3 dents insérées sur l'extrémité antérieure de l'œsophage, ouverture buccale bordée de six lèvres; monodelphie, spicules simples et courts, synlophe avec petites arêtes subégales, dont la pointe est orientée de la droite vers la gauche, selon un axe d'orientation frontal.

Genre type: Inglamidum n. gen.

Espèce type unique: Inglamidum akodon n. sp., parasite d'un Cricétidé chilien, en l'honneur de notre collègue le $\mathrm{D}^{\mathrm{r}}$ W.-G. Inglis.

\section{Bibliographie}

Durette-Desset (M.-C.), 1968. - Helminthes de Marsupiaux américains, Trichostrongyloidea. Bull. Soc. Zool. Fr., 93, 581-594.

Durette-Desset (M.-C.), 1971. - Essai de classification des Nématodes Héligmosomes. Corrélations avec la paléobiogéographie des hôtes. Mém. Mus. Nat. Hist. Nat., $\mathrm{n}^{\text {1le }}$ sér., Série A, Zool., 49, 126 p.

INGLIS (W. G.), 1968. - The geographical and evolutionary relationships of Australian trichostrongyloid parasites and their hosts. J. Linn. Soc. (Zool.), 47, 327-347.

Wertheim (G.) et Durette-Desset (M.-C.), 1976. - Helminthes de Mammifères et d'Oiseaux d'Israël. VI. La taxonomie et l'écologie des Nématodes Trichostrongyloïdes. Ann. Parasitol. hum. comp., 50, 735-762. 